\title{
QCD thermodynamics with two flavours of Wilson fermions on large lattices
}

\section{Bastian B. Brandt* and Anthony Francis}

Institut für Kernphysik, Johannes Gutenberg-Universität Mainz, Johann Joachim Becher-Weg 45, 55099 Mainz, Germany

E-mail: brandt@kph.uni-mainz.de

\section{Harvey B. Meyer and Hartmut Wittig}

PRISMA Cluster of Excellence,

Institut für Kernphysik, Johannes Gutenberg-Universität Mainz,

Johann Joachim Becher-Weg 45, 55099 Mainz, Germany

and

Helmholtz Institut Mainz, Johannes Gutenberg-Universität Mainz,

Johann Joachim Becher-Weg 36, 55099 Mainz, Germany

\section{Owe Philipsen}

Institut für Theoretische Physik, Goethe-Universität,

Max-von-Laue-Str. 1, 60438 Frankfurt am Main, Germany

\begin{abstract}
We explore the phase diagram of two flavour QCD at vanishing chemical potential using dynamical $O(a)$-improved Wilson quarks. In the approach to the chiral limit we use lattices with a temporal extent of $N_{t}=16$ and spatial extent $L=32,48$ and 64 to enable the extrapolation to the thermodynamic limit with small discretisation effects. In addition to an update on the scans at constant $\kappa$, reported earlier, we present first results from scans along lines of constant physics at a pion mass of $290 \mathrm{MeV}$. We probe the transition using the Polyakov loop and the chiral condensate, as well as spectroscopic observables such as screening masses.
\end{abstract}

The 30th International Symposium on Lattice Field Theory

June 24 - 29, 2012

Cairns, Australia

\footnotetext{
${ }^{*}$ Speaker.
} 


\section{Introduction}

The unresolved question about the order of the phase transition connected to chiral symmetry restoration in the chiral limit of two-flavour QCD is the remaining qualitative issue concerning the phase diagram in the $\left\{m_{u d}, m_{s}, T\right\}$ parameter space at zero chemical potential (see [1] for a review). There are two possible scenarios [2,3]: In the first scenario the chiral critical line reaches the $m_{u d}=0$-axis at some tri-critical point $m_{s}^{\text {tric }}$ and the transition at $N_{f}=2$ with $m_{u d}=0$ is of second order. Then the restoration of chiral symmetry belongs to the $S U(2) \times S U(2) \simeq O(4)$ universality class [4]. In the second scenario the chiral critical line never reaches the $m_{u d}=0$ axis and the transition remains first order for all values of the strange quark mass. In [2,3] it was shown that the realisation of one of the two scenarios can be linked to the strength of the anomalous breaking of the $U_{A}(1)$-symmetry at the transition point in the chiral limit. The strength of the anomaly can be probed by looking at correlation functions in scalar and pseudo-scalar channels and the associated screening masses. Despite a number of recent studies aiming to extract information about the order of the transition in the chiral limit $[5,6,7,8]$ no study has sufficient control over the systematic effects.

In these proceedings we present the current status of our study on the topic, first reported in $[9,10]$, using non-perturbatively $\mathscr{O}(a)$-improved Wilson fermions at $N_{f}=2$. We use lattices with a temporal extent of $N_{t}=16$ throughout to suppress discretisation effects, in particular the effect of the explicit breaking of chiral symmetry introduced by the Wilson term. We aim at simulations at several quark masses corresponding to zero-temperature pions with $m_{\pi} \lesssim 300 \mathrm{MeV}$ with three different volumes for each simulation points. This will eventually enable us to perform a scaling analysis with control over the main systematic effects. Here we present results on the transition temperatures, screening masses and on the strength of the anomalous breaking of the $U_{A}(1)$-symmetry.

\section{Setup}

The simulations are done using non-perturbatively $\mathscr{O}(a)$-improved Wilson fermions [11] with two degenerate dynamical quarks and the configurations are generated using DD-HMC [12, 13] and MP-HMC [14] algorithms. For more details on the simulation setup see [9].

To extract information about the order of the transition in the chiral limit by means of a scaling analysis, it is important to control and disentangle the different systematic effects that might distort the scaling properties of the results at finite lattice spacing and volume. Of particular importance for Wilson fermions in this context is the suppression of the explicit breaking of chiral symmetry which shows up as a discretisation effect at finite lattice spacing. Therefore our simulations are done on large lattices of the size of $16 \times 32^{3}, 16 \times 48^{3}$ and $16 \times 64^{3}$. The three different volumes at each simulation point allow for an extrapolation to infinite volume, and at this large temporal extent one can expect discretisation effects to be small (see also [15]).

We scan in the temperature by varying the bare coupling $\beta$ while $N_{t}=16$ remains fixed either at fixed hopping parameter $\kappa$ (for heavier quarks) or along lines of constant physics (for quarks with an associated $m_{\pi} \lesssim 290 \mathrm{MeV}$ ). We set the scale by using an interpolation of the zero-temperature results for the Sommer parameter $r_{0} / a$ in the chiral limit obtained within the CLS effort in the 


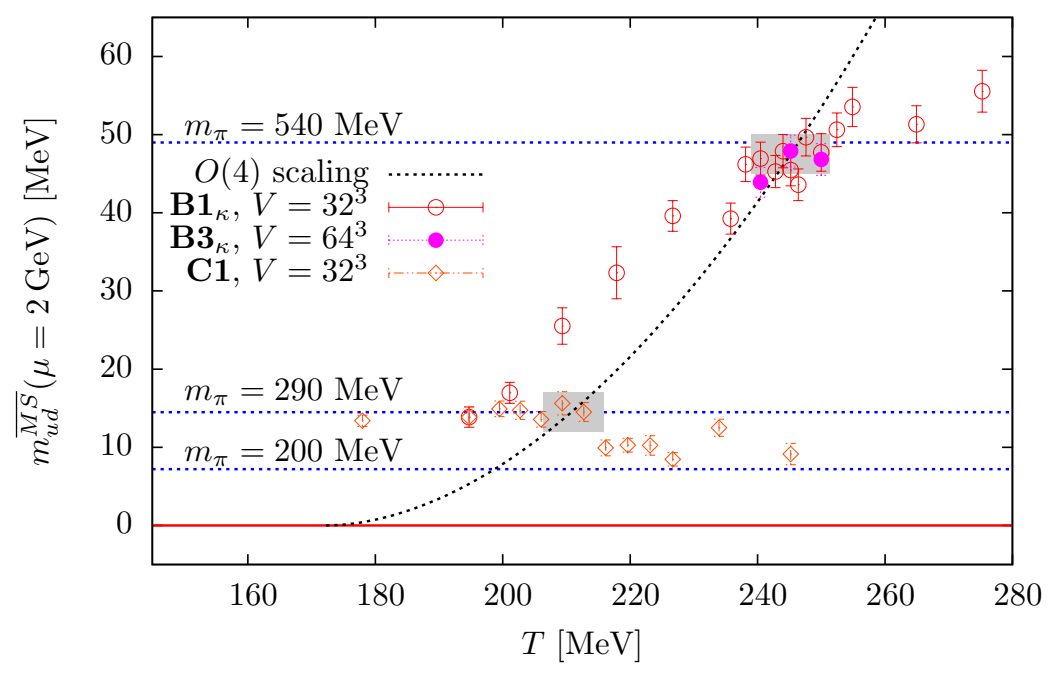

Figure 1: Simulation points in the $\left\{m_{u d}, T\right\}$-parameter space. The black dashed line results from naive $O(4)$-scaling for the two transition temperatures indicated by the grey squares and is only shown to give a first impression on the possible location of the critical line.

region of $5.20 \leq \beta \leq 5.50[16,17]$ and its continuum result $r_{0}=0.503(10)$ fm obtained in [17]. Similarly, the tuning of the bare parameters to lines of constant renormalised quark masses is done using the input from results obtained within CLS [17, 18, 19].

We extract the critical temperature for the deconfinement transition and the chiral symmetry restoration using the real part of the APE-smeared Polyakov loop $L_{\mathrm{SM}} \equiv \operatorname{Re}\left[\left\langle L_{\mathrm{SM}}\right\rangle\right]$ and the subtracted chiral condensate $[20,21]$

$$
\langle\bar{\psi} \psi\rangle_{\mathrm{sub}}=2 \frac{N_{f} T}{V} m_{\mathrm{PCAC}} \int d^{4} x\langle P(0) P(x)\rangle,
$$

respectively. Here $P(x)$ is the pseudoscalar density and $m_{\mathrm{PCAC}}$ the PCAC mass. The transition temperatures are defined by the position of the peak of the associated susceptibilities

$$
\chi(O) \equiv N_{s}^{3}\left(\left\langle O^{2}\right\rangle-\langle O\rangle^{2}\right)
$$

where $O$ is any of the observables above. For the time being all those observables are unrenormalised. The error analysis has been done using the bootstrap method with 1000 bins.

\section{Current status}

The current set of simulation points in the $\left\{m_{u d}, T\right\}$-parameter space ${ }^{1}$ is shown in figure 1 . The set of points consists of two different temperature scans, one at constant $\kappa$ with two volumes (red and magenta circles) and another one at a line of constant quark mass of $m_{u d}=14.5 \mathrm{MeV}$ (orange diamonds). The associated parameters are listed in table 1.

\footnotetext{
${ }^{1}$ Here $m_{u d}$ is the renormalised quark mass in the $\overline{M S}$-scheme at a renormalisation scale of $\mu=2 \mathrm{GeV}$.
} 


\begin{tabular}{c|ccccccc}
\hline \hline scan & Lattice & DD & $\kappa$ & $m_{u d}[\mathrm{MeV}]$ & $T[\mathrm{MeV}]$ & $\tau_{U_{P}}[\mathrm{MDU}]$ & MDUs \\
\hline $\mathbf{B 1}_{\kappa}$ & $16 \times 32^{3}$ & $8^{4}$ & 0.136500 & - & $190-275$ & $\sim 10$ & $\sim 20000$ \\
$\mathbf{B 3}_{\kappa}$ & $16 \times 64^{3}$ & $8 \times 4^{3}$ & 0.136500 & - & $240-250$ & $\sim 46$ & $\sim 16000$ \\
\hline $\mathbf{C 1}$ & $16 \times 32^{3}$ & $8^{4}$ & - & $14.5(45)$ & $175-250$ & $\sim 14$ & $\sim 12000$ \\
\hline \hline
\end{tabular}

Table 1: Scans at $N_{t}=16$ at constant $\kappa$, the ones with subscript $\kappa$, and at constant renormalised quark mass, C1. Listed are the size of the DD-HMC blocks, DD, the temperature range in $\mathrm{MeV}$, the integrated autocorrelation time of the plaquette $\tau_{U_{P}}$ and the number of molecular dynamics units, MDUs, used in the analysis. The measurements have been done each 4 MDUs.

The results for scan $\mathbf{B} 1_{\kappa}$ have in part already been reported in $[9,10]$. The main results remain unchanged even with increased statistics and a larger number of simulation points. The transition temperature in physical units, listed in table 2, changes slightly due to the updated scale determination reported in [17]. Note that the transition temperature is extracted from the peak position of a Gaussian fit to the Polyakov loop or condensate susceptibility peak. The uncertainties are estimated conservatively by the full spread of points included in the fit. The value given in table 2 for scan $\mathbf{B} \mathbf{1}_{\kappa}$ is extracted from the Polyakov loop, but the condensate also shows a (weak) peak in its susceptibility at similar temperature. The additional simulations points from scan $\mathbf{B} \mathbf{3}_{\kappa}$ probe the same transition only with twice the volume. The results are in good agreement with the results for the smaller volume but the limited number of three simulation points does not allow for a reliable determination of the critical temperature. The scan serves as a benchmark for the future simulations on larger volumes.

Scan $\mathbf{C 1}$ is our first scan along a line of constant quark mass of $14.5 \mathrm{MeV}$, which corresponds to a zero-temperature pion mass of about $290 \mathrm{MeV}$, and provides first results in the regime relevant for the future scaling analysis. The results for the smeared Polyakov loop, the subtracted condensate and their susceptibilities are shown in figure 2. As can be seen from the plot, both susceptibilities exhibit a peak at a similar temperature. The result given in table 1 is the one extracted from the condensate, which agrees with the one extracted from the Polyakov loop within errors.

\begin{tabular}{c|ccc}
\hline \hline scan & $T_{C}[\mathrm{MeV}]$ & $m_{u d, C}[\mathrm{MeV}]$ & $m_{\pi, C}[\mathrm{MeV}]$ \\
\hline $\mathbf{B 1}_{\kappa}$ and $\mathbf{B 3}_{\kappa}$ & $245(7)(6)$ & $45(2)(2)$ & $512(30)(15)$ \\
\hline $\mathbf{C 1}$ & $211(5)(3)$ & $14.5(20)(13)$ & $287(25)(13)$ \\
\hline \hline
\end{tabular}

Table 2: Estimates for the transition temperatures, quark and pion masses. The first error bar reflects the uncertainty due to the extraction of the peak position. The second error denotes the uncertainty due to scale setting and renormalisation. For scan $\mathbf{B} \mathbf{1}_{\kappa}$ and $\mathbf{B} \mathbf{3}_{\kappa}$ the result for the transition temperature has been extracted from the Polyakov loop susceptibility, for scan $\mathbf{C 2}$ the value from the subtracted condensate has been used. The conversion from quark to pion masses is done using continuum $\chi$ PT to NNLO [22] with the low energy constants from $[19,23]$. 

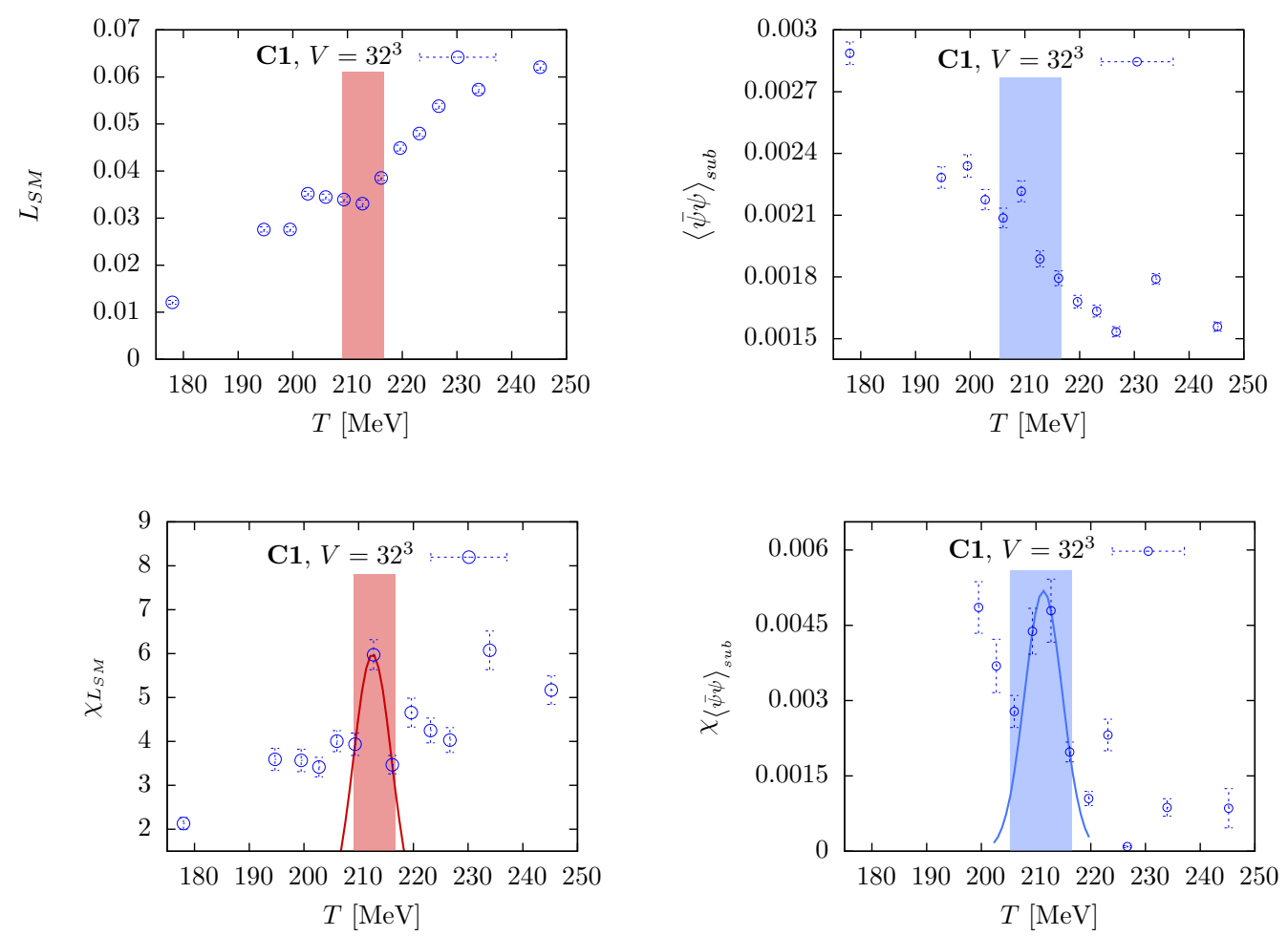

Figure 2: Results for the smeared Polyakov loop $L_{\mathrm{SM}}$ (left) and the subtracted chiral condensate $\langle\bar{\psi} \psi\rangle_{\text {sub }}$ (right) and their susceptibilities for scan C1. The coloured areas are the transition regions extracted from the susceptibility. The curves are the Gaussian fits used to define the transition point.

\section{Screening masses}

Further information about the chiral symmetry restoration pattern in a given scan can be extracted from the behaviour of screening masses [24]. In particular, the degeneracy of pseudoscalar and scalar screening masses signals the restoration of the anomalously broken $U_{A}(1)$-symmetry. In this section we focus on scan $\mathbf{C} 1$ and study screening masses in the pseudoscalar $(P)$, scalar $(S)$, vector $(V)$ and axial vector $(A)$ channels, measured on the stored configurations (separated by 40 MDUs) with a point source.

Figure 3 illustrates the temperature dependence of the screening masses. The $x$-axis is normalised to the critical temperature listed in table 2 . At $0.84 T_{C}$ the screening masses are mostly in agreement with the expected splitting patterns of the zero-temperature meson masses. The screening mass in the pseudoscalar channel, $M_{P}$, starts from a value of $M_{P} /(2 \pi T)=0.33(2)$, which means that at this point $M_{P}$ is a factor of 1.27 larger than the zero-temperature pion mass in the scan. Around $T_{C}$ it then starts to rise and at $1.16 T_{C}$ it is roughly $30 \%$ smaller than the asymptotic value $2 \pi T$. This is in the ballpark of what has been found for the Wilson action on pure gauge configurations [25], but larger than typical results for staggered fermions [26, 27]. Note however, that regardless of the discretisation finite volume (here $N_{s} / N_{t}=2$ ) and quark mass effects might still give a sizeable contribution around $T_{C}$. The $V$ and $A$-channels are accidentally already close to the asymptotic value of $2 \pi T$ below $T_{C}$ and mainly fluctuate around this value in the whole interval. 


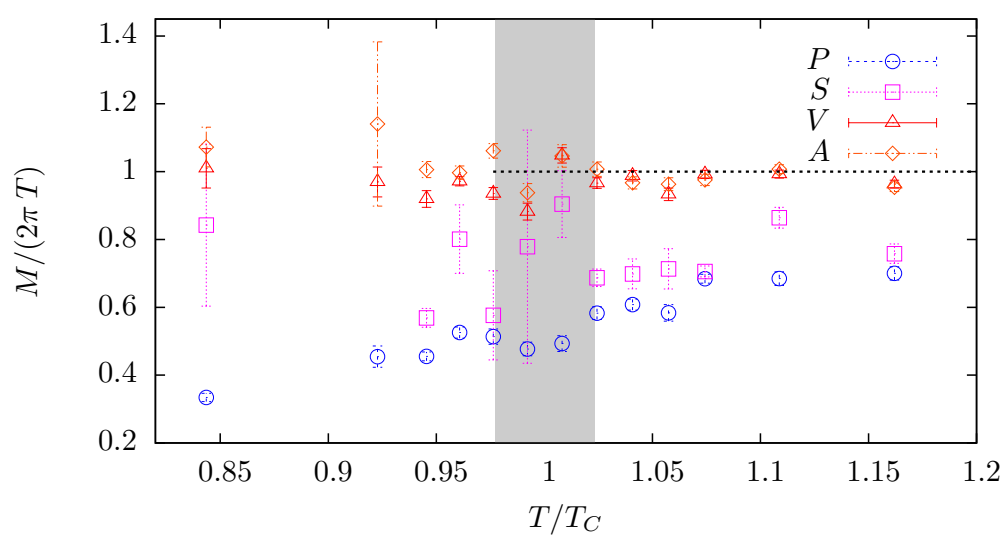

Figure 3: Temperature dependence of the screening masses in $P, S, V$ and $A$ channels. The dashed line corresponds to the asymptotic value of $M=2 \pi T$.

$M_{V}$ and $M_{A}$ fluctuate independently below $T_{C}$ and become degenerate above $T_{C}$, consistent with chiral symmetry restoration.

The strength of the anomalous breaking of the chiral $U_{A}(1)$-symmetry can be assessed via the splitting between the screening masses in $P$ and $S$ channel. Below and at $T_{C}$ the screening mass in the $S$-channel and shows large fluctuations. Above $T_{C}$ the signal becomes more stable and $M_{S}$ moves closer to $M_{P}$, signalling a weakening of the breaking of $U_{A}(1)$. At $1.16 T_{C}$ the symmetry is almost restored. This is in qualitative agreement with the findings from [28] where the symmetry has been found to be restored at about $1.25 T_{C}$.

\section{Conclusions}

This proceedings article contains a summary of the status of our ongoing study of the QCD deconfinement transition in the chiral limit at $N_{f}=2$. To date there are two scans available with $N_{t}=16$ and pion masses at the critical point of about 510 and $290 \mathrm{MeV}$. The critical temperatures are 245 and $211 \mathrm{MeV}$ respectively. The scan at the pion mass of $290 \mathrm{MeV}$ is the first scan in the region important for a future scaling analysis and is done along a line of constant renormalised quark mass of $14.5 \mathrm{MeV}$. To study the pattern of chiral symmetry restoration, we calculate mesonic screening masses. The pseudoscalar screening mass rises from a value close to the zero-temperature pion mass at $0.84 T_{C}$ towards the asymptotic value of $2 \pi T$. At $1.16 T_{C}$ it differs by roughly $30 \%$ from this limit. This is in agreement with the results for screening masses extracted from pure gauge theory with Wilson fermions [25], but larger than typical results found in simulations with staggered fermions $[26,27]$. The results show the expected degeneracy for vector and axial vector channels around and above $T_{C}$, signalling chiral symmetry restoration. At the same time, the $U_{A}(1)$ symmetry still appears to be broken. The large lattices used in our study also offer the possibility to study plasma properties in terms of temporal correlation functions in the vector channel and the associated spectral function (see [29]). A first study has already been reported at conferences and we refer to our future publication for the details. 


\section{Acknowledgments}

The simulations where done on the WILSON cluster at the Institute for Nuclear Physics of the University of Mainz, on the FUCHS cluster at the Center for Scientific Computing of the University of Frankfurt and on JUROPA and JUGENE at FZ Juelich under project number HMZ21. We are grateful to the institutes for offering these facilities. B.B. is supported by DFG Grant ME 3622/2-1.

\section{References}

[1] M. P. Lombardo, plenary talk at this conference.

[2] R. D. Pisarski and F. Wilczek, Phys. Rev. D 29 (1984) 338

[3] A. Butti, A. Pelissetto and E. Vicari, JHEP 0308 (2003) 029 [hep-ph/0307036]

[4] K. Rajagopal and F. Wilczek, Nucl. Phys. B 399 (1993) 395 [hep-ph/9210253]

[5] C. Bonati, et al., PoS LATTICE 2008 (2008) 204 [arXiv:0901.3231 [hep-lat]]

[6] V. G. Bornyakov, et al., Phys. Rev. D 82 (2010) 014504 [arXiv:0910.2392 [hep-lat]]

[7] V. G. Bornyakov, et al., arXiv:1102.4461 [hep-lat]

[8] F. Burger, et al., arXiv:1102.4530 [hep-lat]

[9] B. B. Brandt, et al., PoS LATTICE 2010 (2010) 172 [arXiv:1008.2143 [hep-lat]]

[10] B. B. Brandt, et al., AIP Conf. Proc. 1343 (2011) 516 [arXiv:1011.6172 [hep-lat]]

[11] B. Sheikholeslami and R. Wohlert, Nucl. Phys. B 259 (1985) 572

[12] M. Lüscher, Comput. Phys. Commun. 165 (2005) 199 [hep-lat/0409106]

[13] M. Lüscher, JHEP 0712 (2007) 011 [arXiv:0710.5417 [hep-lat]]

[14] M. Marinkovic and S. Schaefer, PoS LATTICE 2010 (2010) 031 [arXiv:1011.0911 [hep-lat]]

[15] O. Philipsen and L. Zeidlewicz, Phys. Rev. D 81 (2010) 077501 [arXiv:0812.1177 [hep-lat]]

[16] B. Leder, et al. [ALPHA Collaboration], PoS LATTICE 2010 (2010) 233 [arXiv:1012.1141 [hep-lat]]

[17] P. Fritzsch, et al., Nucl. Phys. B 865 (2012) 397 [arXiv:1205.5380 [hep-lat]]

[18] B. B. Brandt, A. Jüttner and H. Wittig, arXiv:1109.0196 [hep-lat]

[19] B. B. Brandt, PhD-thesis, University of Mainz (2012)

[20] M. Bochicchio, et al., Nucl. Phys. B 262 (1985) 331

[21] L. Giusti, et al., Nucl. Phys. B 538 (1999) 249 [hep-lat/9807014]

[22] J. Bijnens, et al., Nucl. Phys. B 508 (1997) 263 [Erratum-ibid. B 517 (1998) 639] [hep-ph/9707291]

[23] B. B. Brandt, A. Jüttner and H. Wittig, in preparation.

[24] C. E. Detar and J. B. Kogut, Phys. Rev. Lett. 59 (1987) 399

[25] S. Wissel, et al., PoS LAT 2005 (2006) 164 [hep-lat/0510031]

[26] M. Cheng, et al., Eur. Phys. J. C 71 (2011) 1564 [arXiv:1010.1216 [hep-lat]]

[27] D. Banerjee, R. V. Gavai and S. Gupta, Phys. Rev. D 83 (2011) 074510 [arXiv:1102.4465 [hep-lat]]

[28] A. Bazavov, et al. [HotQCD Collaboration], arXiv:1205.3535 [hep-lat]

[29] H. -T. Ding, et al., Phys. Rev. D 83 (2011) 034504 [arXiv:1012.4963 [hep-lat]] 